\title{
Introduction: les Amériques et la laïcité
}

Acquis historiques et enjeux actuels

\section{Micheline Milot}

\section{CpenEdition}

Journals

Édition électronique

URL : http://journals.openedition.org/assr/21204

DOI : $10.4000 /$ assr.21204

ISSN : $1777-5825$

Éditeur

Éditions de l'EHESS

Édition imprimée

Date de publication : 1 juin 2009

Pagination : 9-16

ISBN : 978-2-7132-2216-0

ISSN : 0335-5985

Référence électronique

Micheline Milot, "Introduction: les Amériques et la laïcité », Archives de sciences sociales des religions [En ligne], 146 | avril-juin 2009, mis en ligne le 01 juillet 2012, consulté le 20 avril 2019. URL : http:// journals.openedition.org/assr/21204; DOI : 10.4000/assr.21204 


\section{Micheline Milot}

\section{Introduction : les Amériques et la laïcité Acquis historiques et enjeux actuels}

Les Amériques, le Nouveau Monde. Les nombreux pays et territoires de ce vaste continent sont nés des colonisations européennes qui s'amorcent dès le début du $\mathrm{XVI}^{\mathrm{e}}$ siècle, détruisant les systèmes d'organisation sociales indigènes préexistants. Les régimes de relations entre les États et les Églises se sont modulés différemment, selon la capacité des forces sociales ou politiques à se définir à distance des modèles européens et selon les confessions dominantes importées par les colonisateurs, soit l'Église catholique ou les Églises protestantes. Du nord au sud, des problèmes semblables relatifs à la tolérance et à la coexistence ont appelé des solutions qui ont irrigué de diverses manières le processus de laïcisation. Si l'aménagement des libertés religieuses et de l'égalité des cultes ne s'est pas toujours traduit aisément dans le cadre social et juridique, surtout pour l'Amérique latine, la nécessité de la séparation de l'État et de l'Église fut envisagée et se concrétisa dans la majorité des pays plus tôt qu'en Europe. C'est ce qu'avait bien constaté Aristide Briand, l'un des penseurs de la loi de séparation des Églises et de l'État en France. Il écrivait, en 1905: «[1]e régime de la séparation des Églises et de l'État, encore si faiblement et incomplètement mis en pratique en Europe, est, au contraire, largement adopté dans le Nouveau Monde ; le Canada (où une loi de 1854 a sécularisé certains ecclésiastiques et enlevé à l'Église anglicane tout caractère officiel), les États-Unis, le Mexique n'en connaissent point d'autre. On le rencontre encore dans la jeune République de Cuba, dans trois républiques du Centre-Amérique et enfin dans le plus important des États de l'Amérique du Sud : les États-Unis du Brésil » ${ }^{1}$.

Les analyses sur les Amériques, selon un axe continental, sont néanmoins à peu près inexistantes concernant cette problématique, alors même que des travaux comparatifs entre les différents pays d'Europe ${ }^{2}$, entre les États-Unis et la

1. Annexe au Rapport fait au nom de la Commission relative à la Séparation des Églises et de l'État. IV - Législations étrangères, présenté à la Chambre des députés, session 1905.

2. Entre autres, Jean Baubérot, (dir.), Religions et laïcité dans l'Europe des douze, Paris, Syros, 1994, et Françoise Champion, Les läicités européennes au miroir du cas britannique, Rennes, Presses Universitaires de Rennes, 2006. 
France ${ }^{3}$ ou encore entre l'Amérique latine et l'Europe latine ${ }^{4}$ ont donné lieu à des productions scientifiques fort intéressantes. Le présent numéro thématique des Archives des sciences sociales des religions entend combler, du moins partiellement, cette lacune. Une double interrogation traverse toutes les contributions : comment la laïcisation s'est-elle déployée dans les Amériques et quels sont les enjeux liés à ce processus jusqu'à aujourd'hui ? Ce projet se devait toutefois d'être modeste : on ne peut saisir, dans le cadre d'un seul numéro, la diversité religieuse, politique, juridique et culturelle des Amériques, qui comprend cinquantetrois pays et territoires. Il s'agit de donner un aperçu des aménagements laïques qu'on y retrouve en présentant l'analyse de huit contextes nationaux choisis en fonction de configurations historiques et sociales diversifiées, sans prétendre ici à la représentativité géopolitique.

Outre une compréhension des relations entre les États et les Églises ou les religions dans l'axe continental américain, ce numéro comporte une ambition heuristique en ce qui concerne l'usage scientifique de la notion de laicité dans d'autres contextes que celui qui l'a historiquement forgée, la France. Le terme de laïcité se restreint-il à un usage national pour décrire une situation exceptionnelle, ou s'agit-il d'un concept analytique ayant une portée plus universelle ? Dans le domaine des sciences sociales, les questionnements autour de l'universalité des concepts utilisés pour décrire et expliquer des réalités socioculturelles différentes ont cours depuis plusieurs années. La sociologie des religions n'a pas échappé au débat, en particulier en ce qui concerne l'usage des termes de laïcisation et de laïcité, dont on peut se demander s'ils ne sont pas trop teintés d'ethnocentrisme ou d'une perspective strictement occidentale, voire chrétienne. Des études qui élargissent le champ d'observation, en puisant à d'autres disciplines comme les sciences politiques ou juridiques, et qui multiplient les situations analysées, permettent d'évaluer la portée des concepts utilisés et de proposer, le cas échéant, une définition plus théorique que descriptive d'une seule réalité sociopolitique. Quels sont alors les éléments constitutifs de la laïcité qui permettent d'analyser des situations nationales diverses?

Il est indéniable que la notion française de laïcité qui s’est imposée dans les sciences sociales a longtemps reflété, quasi exclusivement, le modèle de relations entre la République française et les cultes. D'application limitée ou restreinte à quelques pays (la Turquie, l'Uruguay ou le Mexique), la notion semblait pour le moins inapplicable au reste de l'Europe et des Amériques. Toutefois, tous les États démocratiques ont mis en œuvre, sous diverses formes, l'autonomie des pouvoirs, la neutralité de l'État, différentes modalités de prise en compte des libertés de conscience, de pensée et de religion et de l'égalité. Une distinction

3. Élizabeth Zoller, (dir.), La conception américaine de la laïcité, Paris, Dalloz, 2005.

4. Jean-Pierre Bastian, (dir.), La modernité religieuse comparée. Europe latine - Amérique latine, Paris, Éditions Karthala, 2001. 
s'impose donc, méthodologiquement, entre ces principes constitutifs de la laïcité et les conditions sociologiques ou terminologiques dans lesquelles ils émergent dans l'aménagement sociétal. Pour les différents auteurs ayant accepté de contribuer à ce numéro, le fait que le terme de laïcité ait d'abord été défini dans la langue française, puis soit demeuré pendant longtemps intimement lié au contexte politique et culturel de l'Hexagone, ne constitue pas un déterminant théorique prohibitif de son usage analytique hors de ce cadre national.

La laïcité correspond à une réalité pluridimensionnelle, à la fois politique, juridique, culturelle et sociale, qui s'est inscrite dans l'histoire des nations au fil des évolutions de la démocratie elle-même. L'État laïque, qui surgit au fil d'un long processus historique, est libre d'élaborer les normes collectives sans qu'une religion ou conviction particulière ne domine ni le pouvoir ni les institutions publiques. L'État tire alors sa légitimité de la souveraineté du peuple et non d'une confession religieuse ou d'une conception philosophique particulière. L'autonomie respective de l'État et des Églises a été constitutionnalisée dans certains pays, tels le Brésil, les États-Unis ou le Mexique. D'autres contrées, dont le Canada, n'ont pas traduit formellement la séparation des pouvoirs dans la constitution, mais divers régimes de neutralité ou de garantie des libertés de conscience, de pensée et de religion se sont graduellement mis en place, ont marqué les théories du droit et ont informé la jurisprudence. À partir de ces prémisses, les configurations de la laïcité peuvent être multiples. Au Mexique et en Amérique latine, par exemple, certains éléments propres à la laïcité se sont développés entre le XVIII et le XIX siècle, c'est-à-dire bien avant l'introduction du concept lui-même (laicidad) dans l'usage juridique ou sociologique. Par ailleurs, dans un pays comme les États-Unis d'Amérique, qui a pratiquement inventé l'idée moderne de séparation entre l'État et les Églises, la notion de Secular State ne recouvre pas du tout ce qui est désigné en français par la sécularisation. Le premier et le quatorzième amendement constitutionnels précisent les principes de séparation et de libertés, alors même que la société ne se trouve pas sécularisée en matière de mours et de morale. À ce propos, Talcott Parsons écrivait, en 1957, qu'il fallait distinguer la sécularisation des valeurs ou des comportements et la sécularisation des structures institutionnelles ${ }^{5}$. La notion de laïcisation permet de définir ce deuxième niveau de manière à ne pas le confondre avec le premier, sinon, il faut alors définir la laïcisation comme faisant partie de la sécularisation ${ }^{6}$. Cette dernière perspective prévaut jusqu'à ce jour, en sciences sociales des religions.

5. Talcott Parsons, "Réflexions sur les organisations religieuses aux États-Unis ", Archives de sociologie des religions, 3, 1957, pp. 21-36.

6. Karel Dobbelaere, par exemple, suggère, dans son article "De la sécularisation ", de parler de laïcisation pour décrire un processus de sécularisation "manifeste » (Revue théologique de Louvain, 39, 2008, pp. 177-196). Voir aussi Jean-Paul Willaime, dans ce numéro. Jorge Ramirez, dans la traduction posthume publiée dans ce numéro, participe de cette compénétration conceptuelle de la laïcisation et la sécularisation. 
Puisque tous les articles dans ce numéro se fondent sur une distinction des deux concepts, celui de la laïcisation et celui de la sécularisation, il nous apparaît pertinent d'expliquer cette perspective.

La sécularisation s'est inscrite au cœur des théories du désenchantement qui décrivent l'effondrement des dispositifs sociaux autrefois sous l'autorité ecclésiale et théologique. La notion a connu une popularité certaine depuis le milieu $\mathrm{du} \mathrm{XX}^{\mathrm{e}}$ siècle dans le champ sociologique. La disqualification culturelle des croyances et des pratiques de la religion conventionnelle dans la plupart des pays modernes, d'une part, le rétrécissement social du champ religieux, d'autre part, ont alimenté pendant plusieurs décennies les thèses de la sécularisation analysant les conséquences de la marginalisation sociale et culturelle de la religion. Les analyses de Peter Berger, Thomas Luckmann, Bryan Wilson et David Martin ont contribué à fournir une architecture théorique au concept de la sécularisation. On ne trouve pas, dans ces analyses, de distinction entre la laïcisation et la sécularisation. Les principes politiques et institutionnels rattachés au concept de laïcisation se trouvent traduits par celui de sécularisation, soit en évoquant les aspects socio-structurels de la sécularisation, soit en différenciant le niveau social du niveau institutionnel. Toutefois, ces auteurs sont davantage préoccupés par les transformations sociologiques de la religion que par les principes politiques et juridiques qu'il revient à l'État d'endosser afin d'assurer le respect des libertés de conscience et l'égalité en cette matière.

L'une des objections souvent formulées à l'encontre de l'usage différencié de la laiicisation et de la sécularisation est le problème d'équivalent dans la langue anglaise. Certes, la traduction du mot apparaît plus tardivement dans d'autres langues que le français, mais la question de la traduction correspond-elle à une problématique empirique, selon laquelle les principes de la laïcité française ne se trouveraient appliqués ou applicables dans aucun autre contexte national ? Il nous semble que, formulé de la sorte, le problème de la traduction est un faux problème. Davantage, certains aspects frappants de l'histoire française (le conflit et l'anticléricalisme) ont très certainement hypothéqué l'usage du mot laïcité dans plusieurs pays des Amériques (et sans doute d'Europe), surtout dans les contextes où l'autonomie des institutions politiques n'a pas nécessité de combattre l'hégémonie d'une Église. Désormais, cette entrave historique semble levée, comme en témoigne l'usage en portugais et en espagnol de laicidad; en Espagne et à Cuba, on retrouve aussi le terme laicismo, qui signifie laïcité et non laïcisme (comme dans le texte original en espagnol du Cubain Jorge Ramirez Calzadilla). En anglais, cohabitent dans les écrits scientifiques l'usage de laicization et de laicity tout comme celui de secularism et de secular. Dans ce numéro, les auteurs étant libres de recourir à la terminologie qui leur est habituelle et selon la langue dans laquelle l'article était rédigé, certains utilisent, en anglais, secularism et secular State, dans le sens strict de laïcisation institutionnelle et d'État laïque, comme chez Roberto Arriada Lorea et Michael J. Perry. D’autres abordent les deux 
réalités, la sécularisation de la société et la laïcisation du politique, dont Roberto Blancarte, Marco Huaco et Micheline Milot, ou encore, laicidad et secularización, chez Juan Esquivel et Nestor Da Costa.

La distinction des deux concepts, laïcisation et sécularisation, apparaît plus éclairante d'un point de vue analytique quand il s'agit d'étudier les relations entre le politique et le religieux et les effets juridiques de ces dernières sur les droits et libertés. Si la sécularisation correspond à une perte progressive de pertinence sociale et culturelle de la religion en tant que cadre normatif orientant les mœurs et les conduites morales « acceptables », la laïcisation évoque davantage la mise à distance institutionnelle de la religion ou des Églises dans la régulation globale de la société. Cette régulation se trouve traduite dans l'univers juridique, de diverses manières. La laïcisation, ainsi entendue, révèle plus nettement que ne le fait le concept de sécularisation non seulement la volonté d'un État de légitimer son action indépendamment des conceptions morales ou religieuses particulières, mais aussi la volonté des organisations religieuses de ne pas subir de contrôle interne de la part du politique. Elle permet également de rendre compte plus adéquatement des principes de droit et de justice qui doivent être mis en œuvre par l'ordre politique dans le cadre d'une démocratie libérale en contexte pluraliste ${ }^{7}$.

La laïcité ne se voit donc pas considérée, dans les différents articles, à partir du modèle français dont il s'agirait de mesurer l'applicabilité. Il ne faut pas entendre par là que les étapes du processus de laïcisation français n'aient eu aucun écho dans les Amériques. Par exemple, l'expérience du Mexique et de l'Amérique latine a pu refléter, du moins par ses caractéristiques générales, le modèle adopté en France, plus précisément celui qui a prévalu sous la domination des jacobins puis celui correspondant à la période de l'instauration de l'éducation laïque, sous la Troisième République. Au Mexique, en Amérique centrale et dans quelques pays de l'Amérique du Sud, comme la Colombie ou le Paraguay, la réalité fut toutefois beaucoup plus complexe parce que le radicalisme républicain eut lieu dès les années 1830, alors qu’à la même époque en France, le modèle concordataire et des cultes reconnus était toujours en vigueur. En d'autres termes, le modèle construit à partir de la Révolution française a servi d'exemple aux républiques latino-américaines, alors même qu'il connaissait en France d'importants revers et reculs au cours du XIX siècle. En retour, les débats entourant la loi de séparation de 1905, en France, n'ont-ils pas fait référence au modèle mexicain, comme le note Briand dans le texte cité plus haut en affirmant : " [l]e Mexique possède ainsi la législation laïque la plus complète et la plus harmonique qui ait jamais été mise en vigueur jusqu’à ce jour ». En réalité, la circulation des idées, des modèles et des conceptions de laïcité a été assez fluide, ce qu'une

7. Voir à ce propos G. Haarscher, La laïcité, Paris, PUF, coll. "Que sais-je ?", 1996, p. 118 et M. Milot, Laïcité dans le Nouveau Monde. Le cas du Québec, Turnhout, Brepols Publishers, 2002, pp. 30-37. 
certaine rigidité théorique pourrait occulter. Elle ouvre ainsi la réflexion théorique à une analyse des synergies entre des courants de pensée et des innovations conceptuelles.

Dans chaque pays étudié, les différentes composantes de la laïcité s'articulent les unes aux autres selon un processus dynamique marqué par l'histoire nationale. Leur mise en œuvre donne lieu à des agencements spécifiques, entremêlés à d'autres valeurs dominantes dans chaque contexte: morale sociale marquée par les normes religieuses, religion civile ou régalisme de l'État, libéralisme philosophique, etc. Il serait vain de tenter de déterminer un modèle de laïcité « américain ", au sens continental. Mieux comprendre les différences entre les processus de laïcisation dans différents pays du Nouveau Monde et leurs effets sociaux, voilà un objectif qui nous paraît plus fructueux. Il s'agit essentiellement de montrer diverses configurations de la laïcisation dans les Amériques, les fondements normatifs à partir desquels elles évoluent et les principaux défis auxquels elles se voient actuellement confrontées. D'où l'intitulé Les laïcités dans les Amériques qui permet d'insister sur la diversité des conceptions sociologiques, juridiques et théoriques en cause.

Outre le contexte historique qui influence le type de régime laïque et son contenu normatif, la laïcité se voit profondément façonnée par le mode de raisonnement éthique prévalant dans chaque société. Le texte de Michael J. Perry, sur les États-Unis, illustre bien ce déplacement par rapport aux modes de raisonnements plus usuels en contexte européen au sujet de la laïité. D’ailleurs, il est frappant de constater qu'aucun auteur n'aborde la problématique de la laïcité par la voie de la nécessaire privatisation de la religion ou celle de l'inadéquation potentielle entre les revendications publiques de l'appartenance religieuse et la laïcité des institutions, deux axes notoires de débats en France. Si l'on cherche un fil d'Ariane reliant les articles rassemblés dans ce numéro, on le trouve aisément dans la préoccupation de débusquer les entraves à l'aménagement d'une laïcité qui puisse assurer la justice et la coexistence harmonieuse. Cette approche critique contribue à mettre en évidence l'intérêt d'examiner plusieurs dynamiques nationales pour mieux évaluer la portée heuristique d'un concept comme celui de la laïcité.

La notion de laïcité recouvre une grande diversité de régimes politiques et d'aménagements sociaux et juridiques des droits et libertés. Certains pays reconnaissent constitutionnellement des Églises d'État, mais il y existe des États où aucune Église n'est établie (en tant qu'institution juridiquement reconnue, représentée par des autorités ecclésiastiques officielles), alors même que la religion, en tant que système de valeurs morales et de doctrines interprétatives de la vie sociale et politique, imprègne les décisions qui émanent des sphères juridiques et politiques. Les constitutions peuvent alors comporter des clauses de séparation, comme au Brésil ou au Mexique, mais l'État n'affiche pas une réelle neutralité envers ce qui a trait à l'égalité juridique et sociale entre les confessions 
et entre des citoyens qui adoptent des conceptions de la vie et de la morale minoritaires. La situation est encore plus évidente au Pérou, comme le décrit bien Marco Huaco qui considère que l'État peut même devenir « pluriconfessionnel », un aménagement selon lequel l'instance politique peut appuyer la légitimité de son action à plus d'une confession, évitant ainsi de s'affranchir complètement de l'Église dominante. Par contre, certains pays ont été historiquement peu préoccupés par l'affirmation juridique de la séparation, mais très attachés aux droits et libertés en matière religieuse, comme le Canada.

Les auteurs ont été invités à se pencher sur la mise en œuvre de la laïcité dans leur pays respectif, à partir des cadres théoriques qui leur sont familiers, qu'ils soient juridiques, sociologiques ou historiques. Là encore, diversité de points de vue et d'approches. Plusieurs questions traversent les études présentées: quelle est la conception dominante de la laicité ? Comment celle-ci se trouvet-elle inscrite dans le droit et traduite par les politiques publiques? Quels sont les droits et libertés garantis aux citoyens et comment ces garanties se traduisentelles concrètement (égalité, non-discrimination, liberté de conscience, etc.) ? À quel point le tissu culturel et social facilite-t-il ou freine-t-il le processus de laïcisation dans l'une ou l'autre des sphères sociales, institutionnelles ou législatives ? Comment le processus de sécularisation interfère-t-il avec celui de la laïcisation?

Ces questionnements soulèvent à leur tour des enjeux théoriques et sociologiques : le traitement juridique ou politique réservé aux minorités, le rôle infrapolitique ou protestataire de certaines Églises ou de groupes de convictions, la portée des clauses de séparation ou de "non-établissement ", l'écart entre les normes constitutionnelles et les comportements socialement "acceptables", comme l'avortement ou l'homosexualité. Un État affichant une indifférence à l'égard des convictions morales et religieuses peut implicitement se montrer hostile à certaines catégories de convictions, ce qui porte atteinte au principe de l'égalité et de la liberté de conscience. Comme la question des droits individuels, mais aussi culturels, prend de plus en plus d'importance au sein des démocraties, quelle est la réponse politique et juridique à celle-ci ? Le pluralisme est-il véritablement intégré dans l'univers politique ou juridique?

La pluralité des situations nationales tout comme la diversité des disciplines à partir desquelles travaillent les auteurs rendent impossible toute tentative d'unifier les approches. Des réalités socioculturelles aussi différentes que les ÉtatsUnis et le Pérou infèrent une perspective analytique elle-même différente. Néanmoins, une certaine convergence des problématiques se fait pressentir autour de la question de la liberté de conscience et de l'égalité, des modalités de leur mise en œuvre et des garanties politiques et juridiques accordées à celles-ci. Les questions des droits humains et de l'adaptation des normes sociales aux valeurs de la modernité libérale se voient davantage freinées par la pesanteur historique de la religion dominante (catholique) en Amérique latine que ce n'est le cas pour le Canada et les États-Unis (où le protestantisme a été prépondérant). Cette 
situation latino-américaine donne lieu actuellement à la formation de nombreux regroupements dans la société civile, revendiquant ce que l'État semble frileux à octroyer, notamment en matière de droits sexuels (avortement, contraception, reconnaissance des droits aux homosexuels, éducation sexuelle à l'école).

Au fil des textes, le lecteur percevra sans nul doute que les auteurs, tout en ancrant leurs analyses dans un espace disciplinaire selon les exigences du travail scientifique, apparaissent également comme des acteurs réflexifs qui entendent participer aux transformations des cadres normatifs de leur espace national, en interrogeant le bien-fondé de ceux-ci pour mieux en montrer le caractère souvent « décalé » par rapport aux requêtes issues de la réalité sociale. Les diverses contributions à ce numéro permettent, à notre avis, une réflexion plus générale sur la laïcité en ce début du XXI ${ }^{e}$ siècle. Elles pourront sans doute inspirer un certain nombre de débats en dehors des axes comparatifs habituels et susciter des analyses élargies à d'autres contextes nationaux.

Micheline MILOT Avec l'aimable collaboration de Roberto Blancarte 